\title{
TRAJETÓRIA DE UM GRUPO DE PESQUISA: PERFIL E PRODUÇÃO CIENTÍFICA*
}

Janislei Giseli Dorociaki Stocco ${ }^{1}$, Karla Crozeta ${ }^{2}$, Aida Maris Peres ${ }^{3}$, Lillian Daisy Gonçalves Wolff ${ }^{4}$, Marineli Joaquim Meier $^{5}$, Maria de Fátima Mantovani ${ }^{6}$

RESUMO: Pesquisa documental, descritiva, de abordagem qualitativa, na qual foram analisadas as produções e publicações dos membros do Grupo de Estudos Multiprofissional em Saúde do Adulto (GEMSA), no período de janeiro de 2005 a junho de 2008, cujos objetivos foram caracterizar as mudanças ocorridas no perfil do grupo e analisar a produção científica desenvolvida pelos seus pesquisadores. As mudanças no perfil do grupo incluem ampliação no quadro de integrantes, reformulações nos objetivos e adequação das linhas de pesquisa. A análise da produção científica denota uma inversão do perfil dos trabalhos produzidos, o que aponta o cumprimento das diretrizes acerca da produção científica. Apreende-se que o GEMSA, ao longo de sua existência, tem favorecido o desenvolvimento de produção de conhecimento científico e de Enfermagem de pesquisadores, docentes, discentes e técnicos ligados à Universidade Federal do Paraná.

PALAVRAS-CHAVE: Enfermagem; Pesquisa; Pesquisa em enfermagem.

\section{TRAJECTORY OF A RESEARCH GROUP: PROFILE AND SCIENTIFIC PRODUCTION}

ABSTRACT: Descriptive, documentary research of qualitative approach, in which the production and publishing materials, from January 2005 to June 2008, from members of the Multiprofessional Study Group in Adult Health (GEMSA) were analyzed. Objectives were: to characterize the changes in the group profile, and to analyze the scientific production developed by its researchers. The changes in the group profile include an increase in the number of researchers, reformulation in the group objectives, and the adjustment of the research line. The scientific production analysis denotes an inversion of the profile in the produced studies, which shows the accomplishment of the scientific production guidelines. It is apprehended that GEMSA, throughout its existence, has being fostering the production development of scientific and Nursing knowledge of researchers, professors, students, and technicians from Federal University of Parana.

KEYWORDS: Nursing; Research; Nursing research.

\section{TRAYECTORIA DE UN GRUPO DE INVESTIGACIÓN: PERFIL Y PRODUCCIÓN CIENTÍFICA}

RESUMEN: Investigación documental, descriptiva, de abordaje cualitativo, en el cual fueron analizadas las producciones y publicaciones de los miembros del Grupo de Estudios Multiprofesional en Salud del Adulto (GEMSA), en el periodo de enero de 2005 a junio de 2008, cuyos objetivos fueron caracterizar los cambios ocurridos en el perfil del grupo y analizar la producción científica desarrollada por sus investigadores. Los cambios en el perfil del grupo incluyen ampliación en el cuadro de integrantes, reformulaciones en los objetivos y adecuación de las líneas de investigación. El análisis de la producción científica denota una inversión del perfil de los trabajos producidos, lo que muestra el cumplimiento de las directrices acerca de la producción científica. Se aprende que el GEMSA, a lo largo de su existencia, ha favorecido el desarrollo de la producción de conocimiento científico y de Enfermería de investigadores, docentes, discentes y técnicos ligados a la Universidad Federal de Paraná.

PALABRAS CLAVE: Enfermería; Investigación; Investigación en enfermería.

*Trabalho produzido na Disciplina de Vivências Integradas de Grupos de Pesquisa do Mestrado Acadêmico em Enfermagem da UFPR. ${ }^{1}$ Enfermeira. Mestranda do Programa de Pós-Graduação em Enfermagem da Universidade Federal do Paraná-PPGENF-UFPR. Membro do Grupo Multiprofissional de Saúde do Adulto-GEMSA.

${ }^{2}$ Enfermeira. Mestranda em Enfermagem pelo PPGENF-UFPR. Bolsista CAPES. Membro do GEMSA.

${ }^{3}$ Doutora em Enfermagem. Professor Adjunto do Departamento de Enfermagem e do PPGENF-UFPR. Orientadora da Disciplina. Vicelíder do Grupo de Pesquisa em Políticas, Gestão e Práticas em Saúde-GPPGPS-UFPR.

${ }^{4}$ Enfermeira. Doutora em Engenharia da Produção. Professor Adjunto do Departamento de Enfermagem e do PPGENF-UFPR, Orientadora da Disciplina. Líder do GPPGPS-UFPR.

${ }^{5}$ Enfermeira. Doutora em Filosofia da Enfermagem. Professor Adjunto do Departamento de Enfermagem e do PPGENF-UFPR. Membro do GEMSA.

${ }^{6}$ Enfermeira. Pós-doutora em Enfermagem. Professor Adjunto do Departamento de Enfermagem e do PPGENF-UFPR. Líder do GEMSA.

Autor correspondente:

Janislei Giseli Dorociaki Stocco

Universidade Federal do Paraná

Av. Iguaçu, 2420 - 80240-030 - Curitiba-PR, Brasil

Recebido: 23/09/09

E-mail: janisleistocco@hotmail.com

Aprovado: 16/12/09 


\section{INTRODUÇÃO}

O trabalho da Enfermagem está condicionado a fatores tecnológicos, políticos, econômicos e sociais, os quais produzem competitividade em um mercado de trabalho especializado. Isso acarreta a necessidade de aperfeiçoamento de habilidades profissionais e a demanda de validação de conhecimentos compatíveis com os avanços tecnológicos e científicos ${ }^{(1)}$.

Essa busca contínua pelo conhecimento sustenta a prática profissional ${ }^{(2)}$ e representa importante estratégia para o fortalecimento da Enfermagem como ciência e profissão. Nesse sentido, a pesquisa se insere na prática e instiga os profissionais à reflexão de seus discursos e ações. Assim, estimula a produção de novos saberes, e é desenvolvida por equipes que compõem os Grupos de Pesquisa, um conjunto de indivíduos organizados hierarquicamente em torno de um, ou eventualmente dois pesquisadores, com experiência, destaque e liderança no âmbito científico ou tecnológico. São compostos pelo líder (que detém a direção acadêmica e intelectual), por pesquisadores, graduados e pós-graduados, e acadêmicos ${ }^{(3)}$.

A organização de Programas de Pós-Graduação Stricto Sensu impulsionou a constituição de grupos de pesquisa na Enfermagem, principalmente na década de 1970, época de crescente aumento de investigações na área, com a produção de teses e dissertações. Esse processo favoreceu a articulação dos saberes, pois os grupos congregam a expertise dos pesquisadores, docentes, enfermeiros e alunos de pós-graduação ${ }^{(4)}$, acrescida à preocupação em inserir a pesquisa na formação profissional, com a participação de discentes da graduação.

Essa articulação favorece os pressupostos dos grupos, com a consolidação das linhas de pesquisa e a validação de abordagens metodológicas que contribuam na publicação do conhecimento produzido e na captação de recursos oriundos de agências de fomento ${ }^{(5)}$. Destaca-se o atual apoio das agências federais ou estaduais de fomento ao desenvolvimento científico e tecnológico, as quais comprovam a necessidade de produzir conhecimentos para a efetivação da Enfermagem como ciência ${ }^{(6)}$.

Nessa perspectiva, os grupos são criados com o intuito de auxiliar na efetivação da pesquisa desenvolvimento e divulgação( ${ }^{(7)}$, institucionalização e agrupamento de interesses de uma mesma área temática, a fim de favorecer a produção científica ${ }^{(8)}$. Assim, foi criado o Grupo de Estudos Multidisciplinar de Saúde do Adulto (GEMSA) da Universidade Federal do Paraná (UFPR), em 1993.

Nessa trajetória de 16 anos, careceu de análise sobre sua articulação com o Programa de PósGraduação em Enfermagem (PPGENF) da UFPR, da produção do conhecimento e das linhas de pesquisa desse programa. Para tanto, restam inquietações acerca das modalidades de organização do trabalho dos pesquisadores, a importância e relevância da produção de conhecimentos, áreas e linhas de pesquisa vinculadas e organização (pessoas, competências/ titulação, fatores que auxiliam ou dificultam a produtividade, políticas de apoio/incremento, perspectivas de avanços) $)^{(2)}$.

O desenvolvimento de estudos que demonstrem a "composição dos grupos da área da Enfermagem, sua vinculação institucional, operacionalização das pesquisas, suas potencialidades, limitações e fragilidades"(2:318), são fundamentais para delinear estratégias de fortalecimento dos grupos de pesquisa brasileiros.

Considerando a implementação do Mestrado Acadêmico em Enfermagem da UFPR, em 2002, as mudanças ocorridas na constituição e na produção científica do GEMSA e a necessidade de estudos acerca dos grupos de pesquisa, objetiva-se caracterizar as mudanças ocorridas no perfil deste grupo e analisar a produção científica desenvolvida pelos seus pesquisadores.

\section{MATERIAIS E MÉTODOS}

Pesquisa documental, descritiva, de abordagem qualitativa, na qual foram analisadas as produções e publicações dos pesquisadores do GEMSA, no período entre janeiro de 2005 a junho de 2008. Os critérios de inclusão neste estudo foram: estar cadastrado no diretório de grupos de pesquisa como 'pesquisador' e manter efetiva participação nas atividades do grupo. Foram incluídos os cinco doutores e um doutorando que compunham o grupo no período da coleta de dados.

Os dados foram encontrados no site do Conselho Nacional de Desenvolvimento Científico e Tecnológico - CNPq, nos Curriculum Lattes dos pesquisadores e provenientes de documentos, livros e resumos de trabalhos do grupo ${ }^{(9-10)}$.

Destacou-se, para caracterização do perfil do grupo, a composição, objetivos e linhas de pesquisa atuais; e para a análise da produção dos pesquisadores foram considerados os projetos de pesquisa, o número 
de orientações resultantes destes, as linhas de pesquisa cadastradas pelos pesquisadores em relação àquelas definidas pelo grupo, e por fim, a produção de artigos contidos no Curriculum Lattes dos pesquisadores.

A análise dos dados seguiu a organização do currículo dos pesquisadores e contemplou as produções bibliográficas relevantes, constituídas de artigos completos publicados em periódicos, trabalhos completos e resumos expandidos publicados em anais de eventos e artigos aceitos para publicação; além disso, considerou-se as orientações (em andamento e concluídas) e as revistas em que o grupo teve maior número de publicações no período de 2005 a 2008.

\section{RESULTADOS E DISCUSSÃO}

O GEMSA foi criado em seis de dezembro de 1993, mediante a inquietação de um grupo de profissionais de várias áreas do saber quanto ao atendimento do adulto nos programas de saúde. Para constituição do seu objetivo, os profissionais basearamse na valorização do indivíduo em todas as suas dimensões, na compreensão do que é 'ser adulto' a fim de ajudá-lo a demarcar seu espaço, definir seus direitos e torná-lo sujeito ativo do processo de cuidar ${ }^{(9-10)}$.

O GEMSA era composto inicialmente por 18 membros: três enfermeiros assistenciais, cinco enfermeiros docentes do Departamento de Enfermagem da UFPR e da Pontifícia Universidade Católica do Paraná, um docente do Setor de Educação, um do Departamento de Estatística da UFPR, um bolsista de aperfeiçoamento, cinco bolsistas de Iniciação Científica e dois bolsistas-permanência ${ }^{(9-10)}$.

Atualmente, possui 50 participantes, sendo: sete pesquisadores pós-graduados, quatro enfermeiros caracterizados como técnicos, 39 estudantes de graduação e pós-graduação (bolsistas de Iniciação Científica, CAPES, Extensão) e acadêmicos voluntários de Iniciação Científica e Extensão. Destaca-se a participação dos enfermeiros, caracterizados como técnicos no diretório de grupos de pesquisa. Parcerias entre profissionais da academia e do serviço são relevantes em projetos de pesquisa, pois unem elementos com experiências distintas e indispensáveis, que facilitam a integração entre a teoria e a prática da Enfermagem ${ }^{(11)}$.

Inicialmente, os objetivos do grupo eram: analisar a demanda de serviços de saúde da população foco do estudo; identificar as principais questões de saúde da população adulta de Curitiba; e planejar ações de suporte para o enfrentamento dos problemas de saúde ${ }^{(9-10)}$. Os objetivos do grupo foram ampliados e reformulados, e atualmente são descritos como: 1) integrar profissionais de diversas áreas do conhecimento para planejar ações que forneçam suporte para o enfrentamento dos problemas de saúde do adulto; 2) possibilitar articulação entre ensino, pesquisa e extensão para melhorar a qualidade de vida da população; 3) divulgar o conhecimento produzido e, 4) viabilizar aos profissionais e estudantes de áreas afins a participação nos projetos de pesquisa e extensão desenvolvidos pelo grupo ${ }^{(9-10)}$.

Denota-se que, com a ampliação na composição do grupo de pesquisa, foram estabelecidos objetivos vinculados à integração dos profissionais das diversas áreas do conhecimento, à articulação entre ensino, pesquisa e extensão e à divulgação da produção científica. Tais aspectos se coadunam com a trajetória dos grupos de pesquisa para o avanço do conhecimento, construção e validação de novas tecnologias, formação e fortalecimento profissional nas diversas áreas de atuação e trabalho ${ }^{(12)}$. Entretanto, manteve-se a preocupação inicial em delimitar estratégias de suporte para o enfrentamento dos problemas de saúde do adulto, elemento que impulsionou a criação do GEMSA.

No processo de ajuste de objetivos e rumos, ocorreu a adequação das suas linhas de pesquisa, juntamente com a implementação do Mestrado Acadêmico da UFPR, aliadas às exigências atuais de produção do conhecimento do programa de pósgraduação(6). Suas linhas de pesquisa eram "Saúde e qualidade de vida"; "O corpo e seus significados" e "Educação em saúde" (9-10). No quadro 1, descrevemse as atuais linhas de pesquisa, as palavras-chave e os objetivos, de acordo com os respectivos números que as identificam na quadro 2.

As linhas de pesquisa estabelecidas na adequação do GEMSA seguem a proposta de agrupamento da produção científica de pós-graduação em Enfermagem, a qual inclui três categorias: profissional, assistencial e organizacional ${ }^{(13)}$. As linhas iniciais do grupo foram inseridas nos itens dois, três e quatro; destaca-se o crescimento com a inclusão da linha um, que se refere a "Processo de Cuidar".

As reformulações dos objetivos e a adequação das linhas de pesquisa foram vinculadas a aspectos relacionados às necessidades da população, realizadas de modo progressivo, a fim de propiciar condições para o engajamento de novos pesquisadores e articulação entre seus integrantes. 
Quadro 1 - Linhas de pesquisa CNPq. Curitiba, 2008.

\begin{tabular}{|c|c|c|}
\hline Linha de Pesquisa & Palavras-Chave & Objetivos \\
\hline $\begin{array}{l}\text { Processo de Cuidar em } \\
\text { Saúde e Enfermagem }\end{array}$ & $\begin{array}{l}\text { Corporeidade; Cuidado; Cuidado de } \\
\text { Enfermagem }\end{array}$ & $\begin{array}{l}\text { Propor e estudar aspectos ligados aos processos } \\
\text { de cuidar, à corporeidade e ao viver saudável }\end{array}$ \\
\hline $\begin{array}{l}\text { Saúde e Qualidade de } \\
\text { Vida }\end{array}$ & $\begin{array}{l}\text { Doença Crônica; Educação em } \\
\text { Saúde; Enfermagem; Qualidade de } \\
\text { Vida }\end{array}$ & $\begin{array}{l}\text { Incentivar projetos para promoção da saúde e da } \\
\text { qualidade de vida de adultos e idosos }\end{array}$ \\
\hline $\begin{array}{l}\text { Fundamentos Teóricos e } \\
\text { Filosóficos do Cuidar em } \\
\text { Saúde }\end{array}$ & $\begin{array}{l}\text { Fundamentos Teóricos e Filosóficos } \\
\text { do Cuidar em Saúde; Corporeidade; } \\
\text { Teorias de Enfermagem e } \\
\text { Tecnologias }\end{array}$ & $\begin{array}{l}\text { Propor estudos teóricos sobre os fundamentos do } \\
\text { cuidar em saúde e enfermagem }\end{array}$ \\
\hline $\begin{array}{l}\text { Políticas e Práticas de } \\
\text { Educação, Saúde e } \\
\text { Enfermagem }\end{array}$ & $\begin{array}{l}\text { Educação em Saúde; Processo de } \\
\text { Trabalho }\end{array}$ & $\begin{array}{l}\text { Identificar e caracterizar a dimensão educativa no } \\
\text { processo de trabalho de Enfermagem;propor } \\
\text { metodologias alternativas e tecnologias opcionais } \\
\text { para educação em saúde }\end{array}$ \\
\hline
\end{tabular}

No tocante à produção científica, buscou-se a relação entre as linhas e os 10 projetos de pesquisa como foco principal, uma vez que "das linhas de pesquisa surgem ou derivam os projetos nas suas diferentes modalidades”(1:317). No entanto, isso não foi possível mediante a inexistência da explicitação da linha a que pertence o projeto, pelo pesquisador. Assim, procedeu-se a análise das linhas de pesquisa definidas pelos pesquisadores em seus currículos e comparadas às linhas cadastradas pelo grupo, conforme quadro 2.

Quadro 2 - Linhas cadastradas pelo pesquisador e no diretório dos grupos de pesquisa CNPq. Curitiba, 2008.

Linhas de Pesquisa

$\begin{array}{lll}\text { Pesquisador } & \begin{array}{l}\text { Cadastradas pelo Pesquisador no Currículo } \\ \text { A }\end{array} & \begin{array}{l}\text { Cadastradas no Diretório dos } \\ \text { Grupos de Pesquisa* }\end{array} \\ \text { B } & * * & 1 \text { e } 2 \\ \text { C Estratégia } & 4 \\ \text { D } & 1,2 & 1 \text { e } 3 \\ \text { E } & 1,2,4 & 1,2 \text { e } 4 \\ \text { F } & 2,4, \text { Fundamentação Tecnológica da Prática Profissional na } & 1 \text { e } 4 \\ & \text { Enfermagem. } & 2 \text { e } 4\end{array}$

*Linha 1 - Processo de Cuidar em Saúde e Enfermagem; Linha 2 - Saúde e Qualidade de Vida; Linha 3 - Fundamentos Teóricos e Filosóficos do Cuidar em Saúde; Linha 4 - Políticas e Práticas de Educação, Saúde e Enfermagem. **O pesquisador não definiu a linha de pesquisa no currículo.

De acordo com o currículo dos pesquisadores, a linha “Saúde e Qualidade de Vida” (linha 2) apareceu no currículo de quatro pesquisadores, seguida da linha "Políticas e Práticas de Educação, Saúde e Enfermagem” (linha 4) inserida em três currículos e, da linha "Processo de Cuidar em Saúde e Enfermagem” (linha 1) considerada por dois pesquisadores. A linha de pesquisa "Fundamentos Teóricos e Filosóficos do Cuidar em Saúde” (linha 3) não foi citada no currículo dos pesquisadores, embora 
esteja cadastrada no Diretório dos Grupos de Pesquisa CNPq para um pesquisador. Verificou-se que um pesquisador não definiu a linha de pesquisa no currículo, a qual foi assinalada com duplo asterisco no quadro 2 .

Consoante à proposta de agrupamento da produção científica de pós-graduação em Enfermagem $^{(13)}$, o escopo das quatro linhas que compõem o grupo está inserido nas três categorias da proposta mencionada; todas estão vinculadas ao cuidado, porém exigiram complementação, uma vez que foram incluídas pelos pesquisadores em seus currículos quatro linhas distintas: "Doenças Infecciosas: Problemática, Especificidade e Estratégias”; “Corporeidade”; "Fundamentação Tecnológica da Prática Profissional na Enfermagem” e, 'História, Cultura e Sociedade’. O termo 'corporeidade', atualmente, deriva-se da linha de pesquisa inicial “O corpo e seus Significados” e está inserido como palavra-chave da linha 3.

As linhas complementares estão em consonância com o setor de aplicação e a árvore do conhecimento que caracterizam o grupo para tanto são suplementares às demais. Percebe-se certa divergência entre a linha cadastrada pelo pesquisador em seu currículo e aquela que o identifica no diretório dos grupos de pesquisa. Além disso, os pesquisadores cadastram mais de uma linha, o que denota "pouca clareza da sua determinação, levando a perceber superposição de linhas, imbricação ou interfaces, concentração ou convergência, pulverização ou diversidade de temas, dentre outros"(2:317).

Assim, sugere-se a necessidade de discussão e padronização das linhas de pesquisa entre os pesquisadores do grupo, bem como a definição de estratégias de delimitação dos temas em relação aos eixos pesquisados, o estabelecimento de projetos amplos que englobem temas específicos e a descrição da linha de pesquisa à qual pertence o projeto, na descrição do mesmo. Questiona-se a possibilidade de exclusão da linha "Fundamentos Teóricos e Filosóficos do Cuidar em Saúde” da estrutura do grupo de pesquisa, uma vez que possivelmente existem, entre os pesquisadores, olhares diferentes sobre objetos de pesquisa das diversas áreas de atuação, quando a finalidade é a mesma e consequentemente poderiam compor a mesma linha de pesquisa.

A análise dos currículos dos cinco doutores e um doutorando que fizeram parte do estudo apontou que houve uma inversão do perfil da produção científica, uma vez que nos anos de 2005 e 2006 o maior enfoque era de trabalhos publicados em eventos.
Em contrapartida, nos anos de 2007 e 2008, foram priorizadas as produções de resumos completos publicados em anais de eventos e artigos publicados em periódicos. Essa inversão aponta o cumprimento das diretrizes da Coordenação de Aperfeiçoamento de Pessoal de Nível Superior (CAPES) no que se refere à produção científica.

O quantitativo de artigos publicados no período estudado foi crescente, com o maior número no ano de 2007 (19 artigos) e nenhum publicado no primeiro semestre de 2008 (apenas três aceitos para publicação). Em relação aos periódicos, percebe-se o predomínio da revista de Enfermagem da UFPR (40\%), no entanto ressalta-se que as publicações desses anos foram encaminhadas a outras revistas.

De acordo com a descrição dos currículos dos pesquisadores, foram realizadas cinquenta orientações (concluídas e em andamento). Porém, os dados disponibilizados pelo Diretório dos Grupos de Pesquisa do CNPq apontam a somatória de setenta e duas orientações concluídas no período estudado. Esta discordância assinala falhas no preenchimento dos currículos e no registro dos dados de cada projeto de pesquisa.

\section{CONSIDERAÇÕES FINAIS}

As mudanças ocorridas no perfil do GEMSA retratam a preocupação em manter o elo e a troca de experiências entre os integrantes das áreas de atuação da Enfermagem. Isso tem contribuído na formação de profissionais preocupados com a pesquisa e, sobretudo, na valorização da consciência crítica e reflexão sobre os discursos e práticas.

Percebe-se o avanço do GEMSA no que tange à ampliação de seus objetivos e adequação das linhas de pesquisa, a fim de atender o número crescente de pesquisadores, profissionais e alunos envolvidos no grupo. A análise das linhas de pesquisa definidas pelo pesquisador, em comparação com as descritas pelo grupo, aponta a complementaridade e a necessidade de discussão coletiva para revisão e reestruturação de tópicos gerais do GEMSA. Além disso, sugere-se que a descrição do projeto de pesquisa contemple a linha a que pertence.

Entre as limitações do estudo, destacam-se a desatualização de alguns currículos e a imprecisão de dados, que justificam as divergências identificadas.

A trajetória do GEMSA, em relação ao seu perfil e produção científica, constitui o reflexo das 
necessidades da comunidade científica da Enfermagem, na produção e divulgação dos conhecimentos produzidos por pesquisadores, docentes, discentes e técnicos da Universidade Federal do Paraná.

\section{REFERÊNCIAS}

1. Carvalho V. Cuidando, pesquisando e ensinando: acerca de significados e implicações para a prática da enfermagem. Rev Latino Am Enfermagem. 2004 Set/ Out;12(5):806-15.

2. Erdmann AL, Lanzoni GMM. Características dos grupos de pesquisa da enfermagem brasileira certificados pelo CNPQ de 2005 a 2007. Esc Anna Nery Rev Enferm. 2008 Jun;12 (2):316-22.

3. Conselho Nacional de Desenvolvimento Científico e Tecnológico - CNPq. Grupos de Pesquisa: saiba mais. [acesso em 2008 Ago 05]. Disponível: http://www.cnpq. br/gpesq/saibamais.htm/.

4. Lopes GT. A trajetória da investigação científica no âmbito da enfermagem. Esc Anna Nery R Enferm. 2002 Abr;6(1):53-62.

5. Leite JL, Neto FRGX, Cunha ICKO. Centro de estudos e pesquisa em enfermagem (CEPEn): uma trajetória de 36 anos. Rev Bras Enferm. 2007 Nov/Dez;60(6): 621-6.

6. Favero L, Camargo TB, Peres AM, Lacerda MR. Histórico e produção de um grupo de pesquisa. Cogitare Enferm. 2009 Jan/Mar;14(1):136-43

7. Guariente MHDM, Zago MMF, Soubhia Z, Berbel NAN, Haddad MCL. Análise de publicação de enfermeiras assistenciais com apoio de um núcleo de pesquisa em enfermagem. Semina: Ciênc Biol Saúde. 2006 Jan/ Jun;27(1):41-56.

8. Peres AM. Reconhecendo a situação da pesquisa em enfermagem na universidade pública. Cogitare Enferm. $2001 \mathrm{Jul} / \mathrm{Dez} ; 6(2): 50-8$.

9. Polack YNS, Kalegari DRG, Jouclas VMG organizadoras. Saúde do adulto: um enfoque multidisciplinar. Curitiba: Pinha; 1997.

10. Polack YNS, Jouclas VMG, Lenardt MH, organizadoras. Caderno de resumos das publicações do GEMSA. Curitiba: GEMSA; 1999.

11. Wolff LDG, Mazur CS, Wiezbicki C, Barros CB, Quadros VAS. Dimensionamento de pessoal de enfermagem na unidade semi-intensiva de um hospital universitário de
Curitiba. Cogitare Enferm. 2007 Abr/Jun; 12(2):171:82.

12. Neves EP, Souza IEO. Pesquisa em enfermagem: buscando resgatar a posição do sujeito que a desenvolve. Texto Contexto Enferm.2003 Jul/Set;12(3):387-93.

13. Carvalho V. Linhas de pesquisa e prioridades de enfermagem - proposta com distinção gnoseológica para o agrupamento da produção científica de pósgraduação em enfermagem. Esc Anna Nery Rev Enferm. 2002 Abr;6(1):145-54. 